\title{
La fin de la tradition cunéiforme et les "Graeco-Babyloniaca"
}

\author{
Stefan M. Maul \\ Freie Universität Berlin - CNRS
}

À l'époque où Homère rédigait l'Iliade et l'Odyssée, en Mésopotamie, on écrivait déjà le cunéiforme depuis 2 millénaires et demi. L'écriture originalement pictographique se développa en une écriture combinant logogrammes et signes syllabiques. Au cours du temps l'écriture fut

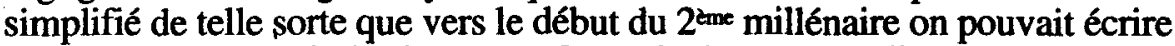
un texte assyro-babylonien avec 80-100 signes. Tandis que la culture cunéiforme extra-babylonienne conservait un système cunéiforme simple, on fait une constatation étonnante à propos des textes littéraires assyrobabyloniens du 1er millénaire av. notre ère. À côté de signes simples du type consonne-voyelle (par ex. $b a, b i, b u$ ) ou bien du type voyelleconsonne (par ex. $a b, i b, u b$ ), les scribes mésopotamiens utilisèrent une multitude de signes du type consonne-voyelle-consonne (par ex. bab ; bar) qui étaient aussi bien d'origine sumérienne qu'akkadienne. On utilisa pour cela en partie des logogrammes désuets ou attestés dans les textes akkadiens avec des valeurs phonétiques qui n'avaient été précédemment utilisées que dans des contextes sumériens. Dans les textes magicoreligieux, divinatoires et médicaux surtout, on abandonna les conventions orthographique simples du vieux babylonien au profit d'une façon d'écrire considérablement plus compliquée. Comme nous le savons par des lettres d'époque néo-assyrienne, les façons d'écrire des textes appartenant aux genres mentionnés ci-dessus pouvaient être si compliquées que la compréhension risquait d'en échapper même à des scribes cultivés de l'époque lorsqu'ils n'étaient point rompus à ces sortes de textes. Aucun document ne le prouve aussi clairement que la lettre suivante qu'envoya à son roi le Chef-Exorciste de la cour du souverain néo-assyrien Esarhaddon (680-669 av. notre ère) :

«Il est vraisemblable que le scribe qui lit au roi ne l'a pas compris [c. à d. une citation de la série ominale Summa izbu $]^{1}$. Summa izbu est difficile à interpréter [c. à d. : «Il est difficile d'en transcrire les logogrammes en Akkadien ?»] Le jour où je serai en présence du roi, mon Seigneur, c'est moi qui, au moyen de cette tablette que j'ai

${ }^{1}$ Dans la série ominale Šumma izbu, qui se compose de 24 grandes tablettes, sont rassemblés tous les présages concernant les avortons de l'homme et des bêtes. Cette série ominale est publiée par E. Leichty, The Omen Series Šumma izbu, Texts from Cuneiform Sources, 4, Locust Valley 1970. 
envoyée au roi, mon Seigneur, montrerai l'entrée ominale (šumu), de la façon dont elle se trouve inscrite. Assurément celui devant qui le doigt (du Maître) n'a pas été suivi tout au long (de la tablette) n'est pas du tout capable de concevoir»².

Dans plusieurs cas on a l'impression que les scribes babyloniens tardifs trouvaient dans l'orthographe un lieu d'élection pour donner un échantillon de leur érudition. En effet quoique le sumérien fût mort, en tant que langue

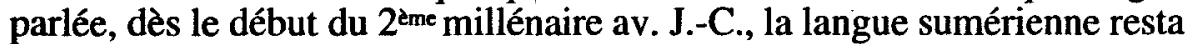
jusqu'à la fin de la culture cunéiforme babylonienne une partie intégrante de la formation des scribes. Dans le culte des Dieux, des Rois et le culte d'Etat, dans les domaines magico-religieux et médical, les textes en langue sumérienne tinrent une place significative même au premier millénaire av. notre ère. Déjà au milieu du $2^{\text {zme }}$ millénaire avant notre ère de tels textes étaient systématiquement pourvus de traductions akkadiennes. Servait pour ce faire un immense corpus suméro-akkadien de listes bilingues avec comparaisons de termes et gloses de prononciation. Ces listes étaient étudiées par les scribes, comme nous l'apprennent les textes scolaires, lors de leur formation. Elles étaient en partie apprises par cœur. Ces listes lexicales bilingues, dont les scribes se servaient comme répertoires de consultations, ne manquent à aucune bibliothèque de tablettes qui disposait de textes littéraires. L'énorme savoir que ces scribes acquéraient dans le cours de leurs études et leur période de formation, influençait presque automatiquement leurs habitudes scribales et les scribes ou érudits utilisaient les listes bilingues comme une source inépuisable pour des associations et des réflexions de nature phonétique qui se concrétisaient dans de nouvelles habitudes orthographiques.

La langue araméenne et avec elle naturellement l'écriture en caractères araméens ont aussi joué en Mésopotamie un rôle plus grand que ne le feraient croire les sources à notre disposition. Cela est montré par la lettre suivante que le roi assyrien Sargon envoya à la fin du $8^{\mathrm{rme}}$ siècle av. notre ère à un de ses serviteurs en Babylonie :

«En ce qui concerne ce que tu m'as écrit: "S'il plaît au roi, que je puisse écrire et envoyer (mes rapports) au roi en araméen et sur des rouleaux en cuir (sipru)", pourquoi n'écris-tu pas et n'envoies-tu pas (tes rapports) sur des lettres (en argile) en akkadien (šipirti akkadâ) ? Assurément, la lettre (en argile) sur laquelle tu écris doit être conforme aux instructions présentes !»?

2 S. Parpola, Letters from Assyrian and Babylonian Scholars, State Archives of Assyria, Vol. X, Helsinki 1993, p. 44 n 60, 15-rev. 14.

${ }^{3}$ Lettre de Sargon à Sîn-iddin de Ur : M. Dietrich, Cuneiform Texts from Babylonian Tablets in the British Museum, Part 54, London 1979, pl. $4 \mathrm{n}^{\circ}$ 10. (La traduction 
Apparemment le roi tenait pour inadmissible que la correspondance officielle entre lui et son serviteur se fit dans une écriture et une langue qui n'étaient point celles héritées de ses prédécesseurs.

Quelques décennies plus tard la réalité avait rattrappé la cour assyrienne. Sur les actes administratifs de l'époque des derniers monarques assyriens Esarhaddon (680-669 av. notre ère) et Assurbanipal (668-627 av. notre ère), lesquels sont toujours en assyrien et en cunéiforme, il n'est pas rare de trouver écrits sur l'argile de la tablette des résumés, au moyen de l'alphabet araméen et à l'encre ${ }^{4}$. Il semble qu'une telle procédure était devenue nécessaire même à la chancellerie royale. Apparemment une partie des fonctionnaires royaux à la cour de Ninive ne maîtrisait plus lè cunéiforme ni l'assyrien et ne pouvait plus se servir que de l'écriture alphabétique araméenne. Lors des fouilles des palais royaux à Ninive, on n'a retrouvé presque que des documents cunéiformes et très peu qui soient écrits en araméen. Cela ne doit pas signifer que l'araméen et, avec lui, les documents écrits en cette langue, ne jouaient qu'un rôle réduit. L'araméen était en effet régulièrement écrit sur du cuir, du papyrus et autres matériaux périssables, qui au contraire des tablettes n'ont pas survécu au temps vu la nature des sols mésopotamiens. Inversement à l'état de choses archéologiques, il faut plutôt penser que l'administration à la cour assyrienne, au ' $7^{\mathrm{eme}}$ siècle avant notre ère, était bilingue et se servait aussi bien de l'alphabet araméen que du cunéiforme. On en a plusieurs indices. Sur les reliefs qui décorent les palais des derniers rois assyriens (et aussi sur des peintures murales ; cf. l'illustration ci-dessous) sont représentés plusieurs scribes qui, pendant une campagne militaire, par exemple, comptabilisent le butin fait par les Assyriens et rédigent des notices à ce propos. Ils sont régulièrement représentés par deux : tandis que l'un inscrit une tablette avec un stylet, l'autre scribe tient en main un support à écriture qui repose sur sa paume et pend vers le bas. Il ne peut s'agir là que d'un rouleau de cuir ou de papyrus. Il est très vraisemblable que ce scribe écrit des notices en langue et écriture araméennes, même si récemment on a proposé que le deuxième scribe fût occupé à des croquis qui devaient servir d'esquisses pour les représentations sur les reliefs de palaiss. Il est significatif qu'à la fin de l'époque néo-assyrienne, outre la désignation traditionnelle du scribe, c'est à dire țupšarru (lídub-sar) qui signifie «l'homme qui écrit la tablette», on en utilise une autre. Cette

suit : S. Parpola, The Correspondence of Sargon II, Part I, State Archives of Assyria, Vol. I, Helsinki 1987, p. XVI).

${ }^{4}$ Cf. F. M. Fales, "Aramaic epigraphs on clay tablets of the Neo-Assyrian Period", Studi semitici, nuova serie 2, Rom 1986.

${ }^{5}$ Cf. J. Reade, Assyrian Sculpture, London 1983, p. 34. Cette interprétation a été rejetée par K. Watanabe, Bibliotheca orientalis 48, 1991, p. 183. 
dernière est "A.BA. Le signe LÚ signifie «homme» et se trouve normalement devant les noms de métiers. On ne peut comprendre qu'on ait choisi la succession A.BA pour désigner le scribe que si l'on admet que $\mathrm{A}$ et BA représentent les deux premières lettres de l'alphabet ${ }^{6}$. C'est comme si aujourd'hui l'on désignait cet homme comme un homme de «l'Alphabet(a)». Il doit donc désigner (au moins primitivement) un scribe qui se servait non pas du cunéiforme mais de l'alphabet araméen.

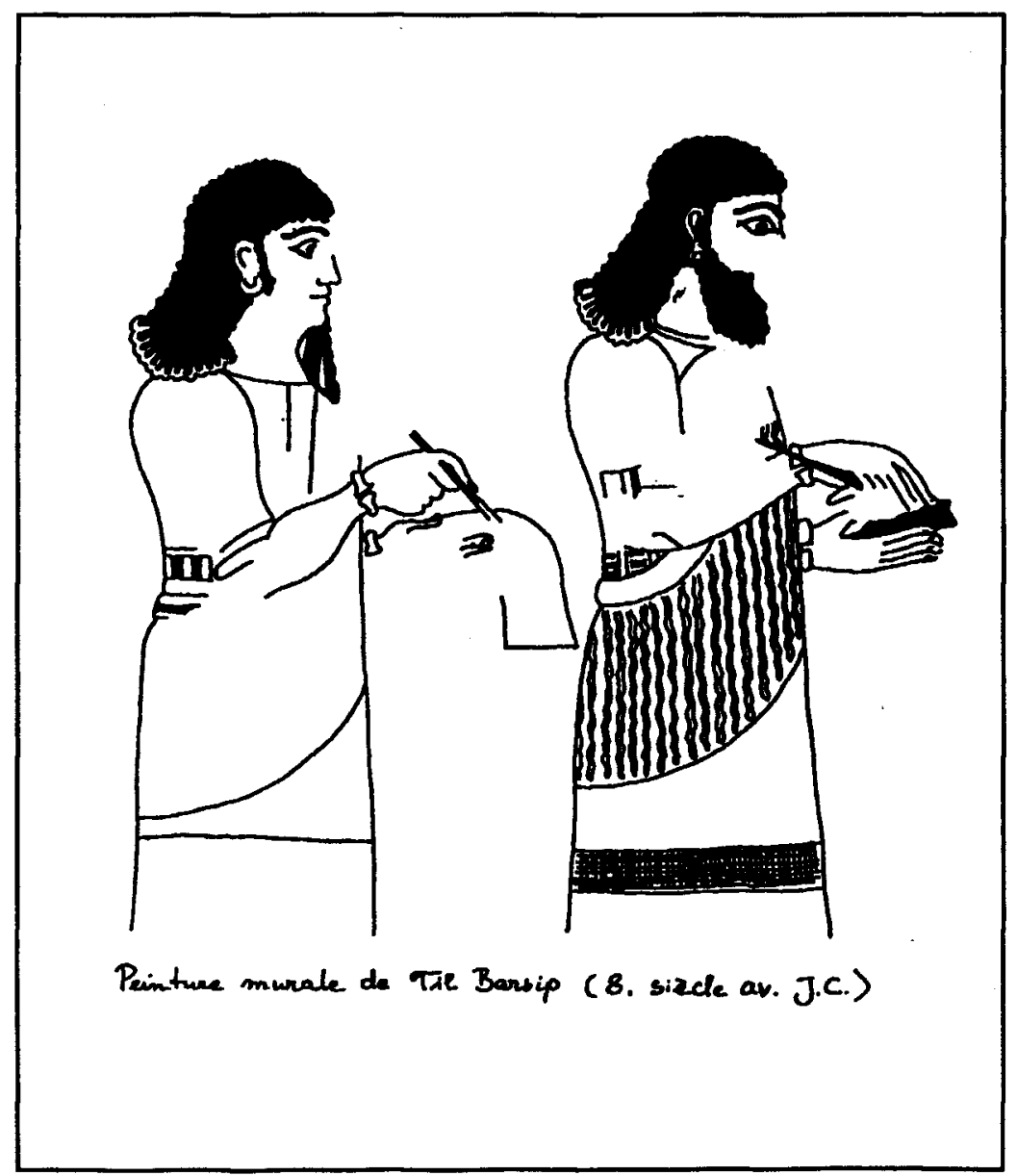

6 Dans les alphabets les plus anciens on retrouve déjà l'arrangement des lettres dans l'ordre que l'on utilise toujours dans l'alphabet hébreu et grec : a b g d etc... 
L'importance grandissante qu'acquiert l'écriture alphabétique araméenne depuis apparemment le début du $7^{\text {èce }}$ siècle avant notre ère dans la vie quotidienne peut avoir été la base de la tendance de l'orthographe cunéiforme, dans les textes littéraires du $1^{\text {er }}$ millénaire avant notre ère, à devenir de plus en plus ésotérique et compliquée et en partie presque cryptique. La compréhension de ces textes était réservée à une petite couche d'érudits de haute culture.

Les rois assyriens avaient renforcé durablement l'extension de la langue et de l'écriture araméennes, sans le vouloir. Surtout aux $8^{\mathrm{emc}}$ et $7^{\mathrm{eme}}$ siècles ils essayèrent de consolider leurs conquêtes dans la zone syro-araméenne en déportant des tribus et en les fixant dans le coeur de l'Assyrie. C'est ainsi que l'araméen s'imposa dans le territoire proprement assyrien. D'un autre côté les échanges intensifs avec l'Égypte conduisirent aussi à ce qu'il se trouve suffisamment de papyrus en Mésopotamie pour concurrencer sérieusement comme support d'écriture les tablettes d'argile. Tout indique donc qu'à partir de la fin de l'époque néo-assyrienne, le cunéiforme fut de plus en plus évincé par l'alphabet araméen plus facile à manier, pour n'avoir plus finalement qu'une survie artificielle due à la conscience de la tradition culturelle babylonienne.

Il est significatif que le roi néo-babylonien Nabuchodonosor et ses successeurs n'aient pas simplement voulu composer leurs inscriptions officielles dans une expression linguistique qui imitât la langue à prestige de l'époque paléo-babylonienne, mais aient aussi utilisé pour elles une graphie en usage vers 2000 avant notre ère, donc environ un millénaire et demi avant eux, tandis que l'araméen avait déjà presque évincé le babylonien en Mésopotamie.

Antiochos-Sôter, lui-même, fils et héritier de Séleucus, le général d'Alexandre, se situa volontairement dans cette tradition. Alexandre avait eu le projet de faire de Babylone la capitale de son Empire et de reconstruire le temple principal de la ville, celui du dieu Marduk, lequel avait été ruiné par Xerxès. Sa mort prématurée à Babylone déjoua cependant ces plans. Antiochos en tant que Anti' ukkus šarru rabâ, «Antiochos le Grand Roi», tout à fait dans la tradition des rois babyloniens, laissa une inscription de fondation cunéiforme en langue babyonienne dans laquelle il parle de sa restauration des temples de Borsippa et de Babylone ${ }^{7}$; il se présente aussi bien sous les traits d'Alexandre que sous ceux d'un Grand Roi babylonien, comme l'avait fait auparavant le roi achéménide Cyrus après sa prise de Babylone. Assurément l'inscription d'Antiochus est le dernier document cunéiforme qu'ait laissé un roi qui ait régné sur la Mésopotamie.

Nous ne connaissons aucune inscription cunéiforme ni pour les successeurs séleucides d'Antiochus ni pour les rois parthes qui ont

7 Cf. F. H. Weissbach, Die Keilinschriften der Achämeniden, Vorderasiatische Bibliothek, Leipzig 1911, p. 132-135. 
contrôlé à partir du milieu du $2^{\text {ème }}$ siècle avant notre ère la Babylonie. On ne connaît pas pour cette époque beaucoup de documents administratifs cunéiformes alors qu'ils sont encore rédigés en grand nombre au $6^{\text {ème }}$ et au début du $5^{\text {tme }}$ siècle avant notre ère. Apparemment l'écriture et la langue grecques avaient largement évincé le cunéiforme. La raison en est sûrement le fait que les derniers Séleucides et les rois parthes se considéraient plus comme des princes hellénistiques que comme les représentants de la Royauté deux fois millénaire de Babylone qui jadis, d'après la tradition suméro-babylonienne, était descendue du ciel. Le cunéiforme lui-même qui était considéré par les Babyloniens comme la marque propre de leur culture, perdit donc la protection que lui avait attribuée la Royauté babylonienne. La langue babylonienne - et par làmême le cunéiforme - avait cessé d'être l'expression des communiqués officiels du Royaume. L'araméen avait la prééminence comme langue vernaculaire et le grec ne servait à la population indigène que comme langue officielle et administrative ${ }^{8}$.

C'est uniquement dans deux domaines qu'on s'attacha encore au cunéiforme à l'époque séleuco-parthe :

- d'une part, les astronomes et astrologues babyloniens que Strabon louait par ailleurs ${ }^{9}$, au tournant du millénaire, pour leurs connaissances extraordinaires, s'en servirent jusqu'au premier siècle de notre ère (le texte cunéiforme datable le plus tardif est de $74 / 75^{10}$ ) ; les astronomes babyloniens observaient et calculaient le cours des astres pour en déduire l'avenir ${ }^{11}$; pour leur dessein le cunéiforme restait le moyen d'écriture le plus signifiant ; pour leurs calculs, le système numéral cunéiforme de position sexagésimale était beaucoup plus simple à manipuler que la notation très peu pratique des chiffres grecs, au moyen de lettres.

- d'autre part, les grands temples de Babylonie, tout particulièrement ceux de Babylone et d'Ourouk, furent le refuge du cunéiforme. Là scribes, lettrés et prêtres copièrent jusqu'au premier siècle avant notre ère hymnes,

8 Cf. J. Oelsner, Materialien zur babylonischen Gesellschaft und Kultur in hellenistischer Zeit, Budapest 1986, p. $245 \mathrm{ff}$.

${ }^{9}$ Strabon, Géographie, XVI 1, 6.

${ }^{10}$ Cf. J. Oelsner, Mitteilungen des Institutes für Orientforschung 17, 1971/72, p. 362 avec note 25 .

" Cf. H. Hunger, A. J. Sachs, Astronomical Diaries and Related Texts from Babylonia, Vol. I : Wien 1988 ; Vol. II : Wien 1989 et en outre l'introduction à l'astronomie babylonienne : B. L. van der Waerden, Erwachende Wissenschaft II. Die Anfänge der Astronomie, Groningen 1966. 
prières, textes magiques et médicaux, conjurations et rituels ${ }^{12}$. Apparemment, on disposait encore à cette époque de fonds de tablettes de bonne qualité et sûres. On a découvert à Babylone une importante bibliothèque avec des tablettes écrites au $2^{\text {eme }}$ et premier siècles avant notre ère et qui contenaient surtout des chants cultuels sumériens ${ }^{13}$. On connaît

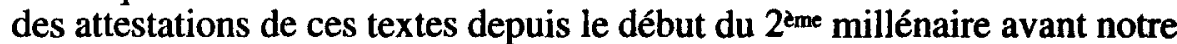
ère. Même si le sumérien était mort comme langue parlée depuis 2000 avant notre ère, ces poèmes avaient toujours été recopiés jusqu'à la fin de la tradition cunéiforme et étaient encore chantés à l'époque séleuco-parthe pour le service divin dans les temples, en l'honneur des dieux. Prêtres et exorcistes apprenaient donc encore au premier siècle av. notre ère le sumérien et s'appliquaient à comprendre, avec l'aide des listes lexicales suméro-akkadiénnes, les textes sumériens et à les pourvoir de traductions babyloniennes, à une époque donc où le babylonien lui-même n'était plus compris et utilisé que par des prêtres et des lettrés.

Les bibliothèques contenant des textes cunéiformes littéraires du temps séleuco-parthe rendent probable, que le prêtre babylonien Bérose qui avait procuré au $3^{\text {zeme }}$ siècle avant notre ère une histoire de la Babylonie en langue

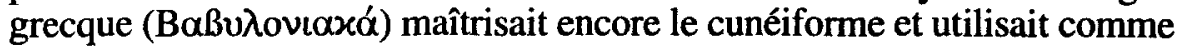
source pour son livre des textes cunéiformes. Il apparaît de plus en plus que les données de Bérose - dont parfois la fiabilité a été mise en doute - sont assurément correctes.

Cependant, même dans les cercles de ces prêtres et lettrés qui, au premier siècle avant notre ère étaient les derniers représentants de la culture babylonienne, l'écriture alphabétique grecque trouva place dans l'usage quotidien.

En même temps que des tablettes d'argile et des chants sumériens, dont l'exemplaire daté le plus tardivement est de 86 avant notre ère ${ }^{14}$, on a trouvé une très remarquable tablette, malheureusement mal conservée, à la fin du dernier siècle. Tandis que sur la face de la tablette est notée une prière en langue babylonienne et en cunéiforme, se trouve sur le revers une transcription de la même tablette, cette fois en écriture alphabétique

12 Cf. J. Oelsner, Materialien zur babylonischen Gesellschaft und Kultur in hellenistischer Zeit, Budapest 1986.

${ }^{13}$ G. Reisner, "Sumerisch-babylonische Hymnen nach Thontafeln griechischer Zeit", Mittheilungen aus den orientalischen Sammlungen 10, Berlin 1896. La deuxième partie de cette bibliothèque sera publiée par l'auteur de cet article dans I. Spar (éd.), Cuneiform Texts in the Metropolitain Museum of Art, Vol. II.

${ }^{14}$ G. Reisner, "Sumerisch-babylonische Hymnen" [cf. note 13], nº 27. 
grecque $^{15}$. Sur la base de critères épigraphiques ce fragment est daté de «sans doute l'époque de Tibère», de 14 à 37 de notre ère. Il appartient donc au groupe des plus récentes tablettes babyloniennes connues. En tout seize fragments de tablettes d'argile (en partie tout petits) contenant des textes sumériens ou akkadiens et dotés d'une transcription écrite en alphabet grec, ont été retrouvés jusqu'à aujourd'hui ${ }^{16}$. Il est probable, que tous ces textes proviennent de Babylone ${ }^{17}$. Quatorze des seize tablettes «gréco-babyloniennes» connues contiennent ou bien contenaient, sur la face un texte cunéiforme et sur le revers une transcription en caractères grecs. Il n'y a que deux textes gréco-babyloniens qui soient exclusivement écrits en écriture grecque. La valeur des textes gréco-babyloniens dont le premier a été découvert en 1902 réside premièrement en ce que les textes écrits alphabétiquement ont tout à fait confirmé la prononciation du sumérien et celle du babylonien postulées par la recherche. Deuxièmement, ces textes sont d'une extrême importance pour la question de savoir jusqu'à quelle époque textes sumériens et babyloniens ont fait l'objet d'une transmission.

En premier lieu, jetons un coup d'œil sur les textes gréco-babyloniens connus. Ils se laissent répartir en deux groupes :

a) Les textes lexicaux

b) Les textes littéraires, surtout les incantations magiques.

La plus grande partie des textes gréco-babyloniens, la plupart du temps conservés de façon très fragmentaire, contient sur la face de la tablette des extraits cunéiformes de listes lexicales suméro-akkadiennes, transmises par les scribes babyloniens depuis plusieurs siècles ${ }^{18}$. Quoique les gréco-

15 Berlin, Vorderasiatisches Museum, tablette VAT 412 ; copie du revers : L. Messerschmidt dans : T. G. Pinches, "Greek Transcriptions of Babylonian Tablets", Proceedings of the Society of Biblical Archaeology 24, 1902, p. 118 ; A.H. Sayce, "The Greeks in Babylonia: Graeco-Cuneiform Texts", ibidem, p. 125 ; photo : E. Schmidt, Archäologischer Anzeiger 1941, 817-118.

${ }^{16}$ E. Sollberger, "Graeco-Babyloniaca", Iraq 24, 1962, p. 63-72 ; M. J. Geller, "More Graeco-Babyloniaca", ZA 73, 1983, p. 114-121 ; J. A. Black, S. M. Sherwin-White, "A Clay Tablet with Greek Letters in the Ashmolean Museum, and the «GraecoBabylonian Texts»", Iraq 46, 1984, p. 131-140 ; S. M. Maul, "Neues zu den «GraecoBabyloniaca»", ZA 81, 1991, p. 87-107.

${ }^{17}$ Cf. E. Sollberger, $\operatorname{lraq} 24,1962$, p. 63.

18 Dans le corpus des græco-babyloniaca on retrouve des extraits des syllabaires $\left(\mathrm{S}^{\mathrm{b}}\right)$, des tablettes $\mathrm{n}^{\circ}$ II et III de la série lexicale HAR-ra = bubullu (cf. E. Sollberger, Iraq 24, 
babyloniaca qui contiennent des textes lexicaux soient en petit nombre, il est facile de constater que les textes conservés correspondent très exactement au domaine d'érudition qu'un élève-scribe devait dominer à l'époque néo-babylonienne. Dans les textes scolaires néo-babyloniens publiés dans OECT $11^{19}$ et MSL SS $1^{20}$ en provenance de Kish, on trouve parmi les extraits lexicaux majoritairement des sections de la série lexicale HAR-ra = hubullu, presque exclusivement en provenance des tablettes I, II et III. Isolément on trouve aussi des extraits d'autres listes comme les syllabaires que l'on appelle $S$ (yllabaire) et $S^{b}{ }^{21}$. Les textes grécobabyloniens contiennent eux aussi des extraits de HAR-ra = hubullu II et III aussi bien qu'un extrait de $S^{b}$. L'apprentissage de ces listes qui étaient d'une importance vitale pour les scribes mésopotamiens à l'époque où le sumérien était mort comme langue parlée, pour la compréhension des prières et conjurations sumériennes, était depuis des siècles une partie effective de la formation. Les élèves-scribes devaient apprendre ces listes par cœur et les recopier sans cesse. Une grande partie des exemplaires aujourd'hui conservés de ces listes lexicales cunéiformes sont d'ailleurs des textes scolaires, donc des exercices d'écriture d'élèves qui apprenaient le cunéiforme et le sumérien. Dans certaines catégories de ces tablettes d'exercice d'époque ancienne on trouve, tout comme dans les textes grécobabyloniens, le même extrait sur face et revers. Les deux versions sur ces tablettes sont cependant en cunéiforme : la première toutefois correcte et dans une belle écriture régulière, la seconde par contre dans un ductus gauche, comportant souvent de grosses erreurs. Une telle constatation ne peut mener qu'à une seule conclusion : c'est le maître qui avait écrit la face de la tablette et l'élève qui avait répété sur le revers, de mémoire, le passage écrit par le maître.

Cette pratique didactique ancienne s'était maintenue apparemment jusqu'au premier siècle de notre ère, dans les écoles de scribes babyloniennes. Seulement les élèves écrivent, sur le revers de la tablette, le passage nouvellement appris et écrit en cunéiforme vraisemblablement par leur maître, non plus en cunéiforme mais dans l'écriture alphabétique grecque réellement plus facile à apprendre. Du fait cependant que la face de la tablette est toujours écrite en cunéiforme, on doit déduire que les élèves

p. 64-67) et de petits fragments d'autres listes lexicales que l'on n'arrive plus à identifier (cf. E. Sollberger, Iraq 24, p. 71-72).

19 O. R. Gurney, Literary and Miscellaneous Texts in the Ashmolean Museum, Oxford Editions of Cuneiform Texts, Vol. 11, $\mathrm{n}^{\circ}$ 103-141.

${ }^{20}$ M. Civil, O. R. Gurney, D. A. Kennedy, Materials for the Sumerian Lexicon, Supplement Series 1, Roma 1986, p. 45ff et pl. I-XXVII.

${ }^{21} \mathrm{Ce}$ groupe de texte joue aussi un grand rôle dans les textes scolaires néo-babylonien retrouvés à Babylone même : cf. A. Cavigneaux, Textes scolaires du temple de Nabû ša Harê, Baghdad 1981 et S. M. Maul, Bibliotheca orientalis 48 (1991), p. 852-860. 
qui avaient recopié en lettres grecques les passages choisis possédaient au moins une connaissance passive du cunéiforme, mais n'avaient à reproduire que le phonétisme du paragraphe appris par eux. L'écriture grecque connue à Babylone grâce aux Grecs qui y exerçaient désormais le pouvoir y convenait beaucoup mieux que l'écriture alphabétique araméenne qui comme presque tous les alphabets sémitiques ne notait les voyelles que de façon insuffisante ou pas du tout. Alors que pour la langue sémitique qu'était le Babylonien, elle pouvait fournir une écriture appropriée, pour la notation du sumérien elle était totalement inadéquate car dans cette langue une grande partie des mots était monosyllabique et la non-notation d'une voyelle dans une langue par ailleurs riche en homophones aurait conduit à une source de confusions sans fin. La question de savoir si les élèves apprenaient aussi à maîtriser de façon active le cunéiforme à l'époque où se produisaient les transcriptions grecques est malheureusement sans réponse. Dans tous les cas, à toutes époques de la culture mésopotamienne, la transmission orale des textes (à côté de la transmission écrite) a joué un grand rôle. Un texte, dans lequel des instructions sont données à un élève, nous apprend ce que l'on attendait d'un élève bien formé : à la fin de sa formation il devait savoir par cour au moins trente à cinquante conjurations et hymnes différents, parmi lesquels certains devaient être très longs ${ }^{22}$.

À côté de listes lexicales, on a gardé quelques textes littéraires qui ont été écrits dans l'alphabet grec. Il s'agit

- d'une liste, souvent copiée dans les écoles scribales, d'épithètes laudatives de Babylone ;

— de deux conjurations bilingues suméro-akkadiennes ;

- d'une prière en babylonien

- et d'un autre texte sur lequel je reviendrai plus tard.

Tout comme en ce qui concerne les listes lexicales, il devait s'agir là de textes scolaires. Des textes de la sorte apartenaient déjà à l'époque néobabylonienne au domaine d'érudition des élèves-scribes.

Qui étaient donc ces élèves ? Étaient-ils grecs ou babyloniens ? Comme les textes gréco-babyloniens appartiennent tout entiers à la tradition conventionnelle des textes scolaires babyloniens, on doit en conclure que les étudiants de ces textes étaient des élèves babyloniens ${ }^{23}$.

Cette façon de voir est étayée par une tablette qui est entièrement écrite en alphabet grec $^{24}$. La tablette contient une prière qui est tout à fait

22 Cf. J. J. A. van Dijk, Orientalia Nova Series 58, 1989, p. 449-452.

${ }^{23}$ Dans $M I O 17$, p. 357-356 [cf. note 10] J. Oelsner a déjà contredit l'opinion de E. Sollberger (Iraq 24, p. 63) selon laquelle ces élèves étaient des étudiants grecs.

${ }^{24}$ W. G. Schileico, "Ein babylonischer Weihtext in griechischer Schrift", Archiv für Orientforschung 5, 1928/29, p. 11-13. 
semblable aux prières des colophons de tablettes néo-babyloniennes ${ }^{25}$. Le propriétaire de la tablette, comme c'était déjà courant à l'époque néobabylonienne, en avait apparemment fait l'offrande à un temple. Il donne

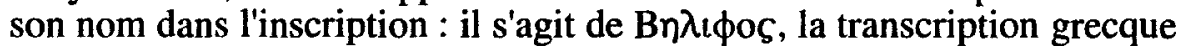
du nom babylonien Bẽl-īpuš. Il est en outre intéressant que soit mentionné

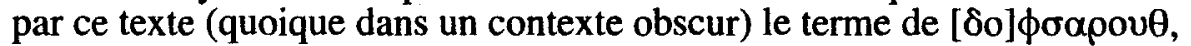
«Science d'écriture des tablettes», en babylonien tupšarrūtu. La mention d'un tel terme rend très vraisemblable que cette tablette écrite uniquement en caractères grecs appartenait à une école scribale babylonienne traditionnelle.

Tous les autres textes gréco-babyloniens ont un modèle cunéiforme. Ce texte est par contre vraisemblablement une nouveauté qui montre qu'au début de notre ère la langue babylonienne était encore pratiquée de façon active par des étudiants babyloniens et, d'un autre côté, que des textes babyloniens n'étaient pas obligatoirement d'abord écrits en cunéiforme.

Â ce moment de l'exposé, nous devons nous souvenir que, vu la nature du sol mésopotamien, les matériaux périssables - au contraire de ce qui s'est passé en Égypte - n'ont généralement pas été conservés. Cela signifie que tous les supports d'écriture en matière organique comme le bois, le cuir ou le papyrus, ont été perdus. En fait, les inscriptions araméennes, par exemple, ne se sont conservées que lorsqu'elles étaient inscrites sur pierre ou argile ou portées sur l'argile grâce à de l'encre. En règle générale cependant elles étaient écrites sur des supports périssables, dont nous connaissons certes l'existence mais qui n'ont pas survécu au temps.

Pourquoi donc ne devrait-il y avoir nulle transcription grecque de textes primitivement cunéiformes qui étaient écrits sur papyrus, pierre ou cuir ? écrire la transcription grecque d'un texte sumérien ou akkadien sur une tablette d'argile n'a en définitive de sens que lorsque l'on note en même temps le texte en cunéiforme! Si un scribe voulait simplement noter une transcription en grec d'un texte babylonien il ne devait certainement pas recourir à un stylet et à de l'argile, un matériau totalement inadéquat pour les signes arrondis de l'alphabet grec $^{26}$, mais utiliser régulièrement les matériaux périssables comme le cuir, le bois, le parchemin ou le papyrus.

25 Cf. par exemple les colophons des tablettes H. H. Figulla, Cuneiform Texts from Babylonian Tablets in the British Museum, Part 42, London 1959, $n^{\circ} 24$ et G. Reisner, "Sumerisch-babylonische Hymnen" [cf. note 13], $\mathrm{n}^{\circ} 56$ (les deux tablettes viennent de Babylone).

$26 \AA$ ma connaissance uniquement deux tablettes en argile qui contiennent des inscription en langue et écritures grecque ont survécu : cf. J. Oelsner, Materialien [cf. note 8] p. 253, 5 avec la note 926 ; F. Cumont, Mémoires archéologiques de Perse XX (1928), p. 97 et pl. IV n 4 (cette tablette à été retrouvé à Suse avec d'autres tablettes écrites en cunéiforme !). 
D'ailleurs, la grande majorité des documents écrits de la vie quotidienne en Babylonie, au début de notre ère, était depuis longtemps notée avec de l'encre sur des matériaux périssables. Nous savons en outre que, déjà au premier millénaire avant notre ère, même des textes cunéiformes étaient inscrits pour une part non négligeable sur des tablettes de cire ${ }^{27}$, qui sauf exceptions rarissimes, n'ont pas été conservées ${ }^{28}$.

Il n'est donc nullement invraisemblable que la transmission de textes sumériens et babyloniens ait perduré après le premier siècle de notre ère, alors que l'on n'inscrivait plus de tablettes d'argile. Toujours est-il qu'à l'époque hellénistique on a recouru à la notation en écriture alphabétique grecque pour d'autres langues et d'autres systèmes d'écritures. Ainsi en Egypte, la vieille écriture égyptienne fut-elle abandonnée, alors que la langue a survécu jusqu'à l'époque actuelle en écriture grecque (avec des signes supplémentaires) sous la forme du «copte». On peut d'autre part se référer aux ' $E \xi \alpha \pi \lambda \tilde{\alpha}$, une édition critique de l'Ancient Testament du père de l'église Origène (185-254 de notre ère) : dans cette édition sextuple, Origène donne non seulement le texte original hébraïque avec quatre traductions grecques différentes mais aussi une transcription du texte hébreu en caractères grecs.

La transmission des textes mésopotamiens et des langues sumérienne et babylonienne sous la forme de transcriptions grecques a donc vraisemblablement duré plus longtemps que la transmission du cunéiforme, si l'on entend par là l'habitude d'utiliser des tablettes d'argile comme support d'écriture. De nombreuses bulles d'argile d'époque héllénistique ont été retrouvées dans plusieurs villes de Babylonie et scellaient primitivement des actes juridiques sur cuir ou papyrus. Ces

27 Vgl. D. J. Wiseman, "Assyrian Writing Boards", Iraq 17, 1955, p. 3-13 ; E. Klengel-Brandt, "Eine Schreibtafel aus Assur", Altorientalische Forschungen 3, 1975, p. 169-171 et pl. 21-22 ; en outre J. Oelsner, MIO 17 [cf. note 10], p. 360 avec note 18 ; O. Pedersén, Archives and Libraries in the City of Assur, Vol. II, Uppsala 1986, p. 43 note 8 et p. 145.

${ }^{28}$ Quelques tablettes trouvées dans la bibliothèque royale d'Assurbanipal à Ninive portent des colophons en caractères cunéiformes écrits à l'encre (cf. J. Reade, "Archaeology and the Kuyunjik Archives", dans: K. Veenhof (éd.), Cuneiform Archives and Libraries, Leiden 1986, p. 217-218; S. M. Maul, "Zukunftsbewältigung", Baghdader Forschungen 18, Mainz 1994, p. 255). Cela montre bien qu'il y avait à l'époque néo-assyrienne des scribes qui maîtrisaient l'art d'écrire des textes en cunéiforme à l'aide d'un pinceau et de l'encre. Peut-être ne se servait-on pas seulement de cet art pour doter d'inscriptions des peintures murales etc..., mais aussi pour noter des textes en cunéiformes sur des supports d'écriture périssables. De toute façon il est remarquable que dans un rituel babylonien l'exorciste devait écrire une incantations sur les ongles des son patient (cf. E. von Weiher, Spätbabylonische Texte aus Uruk, Teil III, Mainz 1993, $\left.\mathrm{n}^{\circ} 81,25-29\right)$, certainement pas avec un stylo, mais avec un pinceau et de l'encre. 
textes ne doivent pas avoir été forcément rédigés en araméen ou en grec ; ils ont pu l'être aussi en langue akkadienne quoiqu'en écriture grecque ${ }^{29}$. Ajoutons que le monde hellénistique auquel le prêtre Bérose, originaire de Babylone, avait procuré au $3^{\text {zme }}$ siecle avant notre ère une histoire événementielle et culturelle de la Babylonie tout à fait fiable $(B \alpha b u \lambda \omega v t \alpha x \alpha)$, avait pour la Babylonie et son histoire culturelle un intérêt sans doute plus fort qu'on ne le pense communément. Ce ne sont point uniquement les sciences hellénistiques de la nature qui ont été influencées d'une façon considérable par des modèles babyloniens. La magie babylonienne et les procédés divinatoires utilisés en Babylonie ont durablement influencé et intéressé le monde hellénistique. Les "Chaldéens", comme on nommait alors les prêtres et les exorcistes babyloniens, ont eu dans le monde gréco-romain la réputation d'avoir pour utiliser les termes mêmes de Diodore (premier siècle avant notre ère) - un «bonheur particulier» dans la science de la prédiction de l'avenir ${ }^{30}$. Dans sa Bibliothèque, Diodore donne une description si détaillée des activités et des domaines d'action des Chaldéens qu'il avait sûrement à sa disposition de bonnes sources fiables à leur propos.

Cicéron, lui-même, n'a pas omis dans son célèbre De divinatione de mentionner la science des Assyriens et des Babyloniens ${ }^{31}$. De même, le poète Théocrite (1 $1^{\text {zre }}$ moitié du $3^{\text {żme }}$ siècle avant notre ère) donne la description d'un charme d'amour babylonien identique à ce qu'on en connaît d'après le recueil de recettes babyloniennes cunéiformes pour recouvrer la puissance sexuelle (šà-zi-ga) ${ }^{32}$. S'il y avait encore à l'époque hellénistique des transcriptions de conjurations babyloniennes, de présages etc..., cette situation serait facile à expliquer, tout comme le fait que les papyrus magiques grecs d'époque hellénistique que $\mathrm{K}$. Preisendanz a publiés ${ }^{33}$, trouvent des parallèles étonnants dans les textes magiques et les conjurations babyloniens, mentionnant aussi bien des dieux babyloniens. La connaissance de textes babyloniens, après le tournant du millénaire

${ }^{29}$ Ainsi J. Oelsner, MIO 17, p. 363.

${ }^{30}$ Diodore, Bibliothèque, II, 29,3.

${ }^{31}$ Cicéron, De divinatione I $2 ; 90-91 ; 93$; aussi II 97.

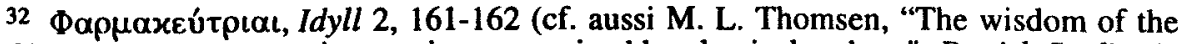
Chaldeans. Mesopotamian magic as conceived by classical authors", Danish Studies in Classical Archaeology, Acta Hyperborea 1, Copenhagen 1988, p. 94). Cf. aussi l'édition des recettes babyloniennes pour recouvrer la puissance sexuelle : R. D. Biggs, ŠA.ZI.GA. Ancient Mesopotamian potency incantations, Text from Cuneiform Sources 2, Locust Valley 1967.

${ }^{33}$ K. Preisendanz, Papyri Graecae Magicae. Die griechischen Zauberpapyri. Zweite verbesserte Auflage, Stuttgart 1973/74 (1. Auflage : Stuttgart 1928/31). 
encore, pourrait fournir une explication du fait qu'un compendium de présages sur les tremblements de terre qui nous est connu depuis le milieu du $2^{\text {zme }}$ millénaire avant notre ère à Nuzi et a été plus tard repris dans la série ominale babylonienne Enūma Anu Enlil, a été ultérieurement

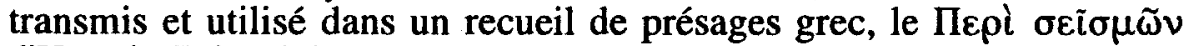
d'Hermès Trismégiste ${ }^{34}$.

On ne doit donc plus s'étonner des allégations d'un roman qui se passe à Babylone, dû à Jamblique lequel vivait à la cour du roi de Grande Arménie Sohaimos (164 après le Christ). Ce roman nous est connu par des extraits de Photius dans sa Bibliothèque. Selon des indications autobiographiques qu'il a inclues dans son roman, Jamblique, fils de parents syriens, aurait appris auprès d'un Babylonien, tombé en esclavage lors de la campagne de Trajan en Mésopotamie, la langue babylonienne, les façons de vivre babyloniennes et les traditions ( $\lambda$ ó $\gamma \circ \iota$ ) babyloniennes $^{35}$. Peut-être des textes babyloniens en écriture grecque étaient-ils encore à la disposition du lexicographe Hésychius d'Alexandrie au $5^{\text {tome }}$ ou $6^{\text {tme }}$ siècles après notre ère, car dans son Lexique, il donne des gloses pour des mots sumériens et akkadiens qui sont tout à fait acceptables ${ }^{36}$.

${ }^{34}$ Cf. C. Bezold et F. Boll, Reflexe astrologischer Keilschriften bei griechischen Schriftstellern, Sitzungsberichte der Heildelberger Akademie der Wissenschaften 1911/VII.

${ }^{35}$ Cf. G. J. F. Gutbrod, "Über die wahrscheinliche Lebensdauer der assyrischbabylonischen Sprache", Zeitschrift für Assyriologie 6, 1891, p. 26-33, notamment p. 32-33 [avec la version originale].

36 Gloses rassemblées par P. Schnabel dans: P. Schnabel, Berossos und die babylonisch-hellenistische Literatur, Leipzig/Berlin 1923, S. 169-171 (Schnabel pense que Hésychius avait connaissance des mots babylonien grâce au livre de Bérose). 
La fin de la tradition cunéiforme et les Graeco-Babyloniaca

17

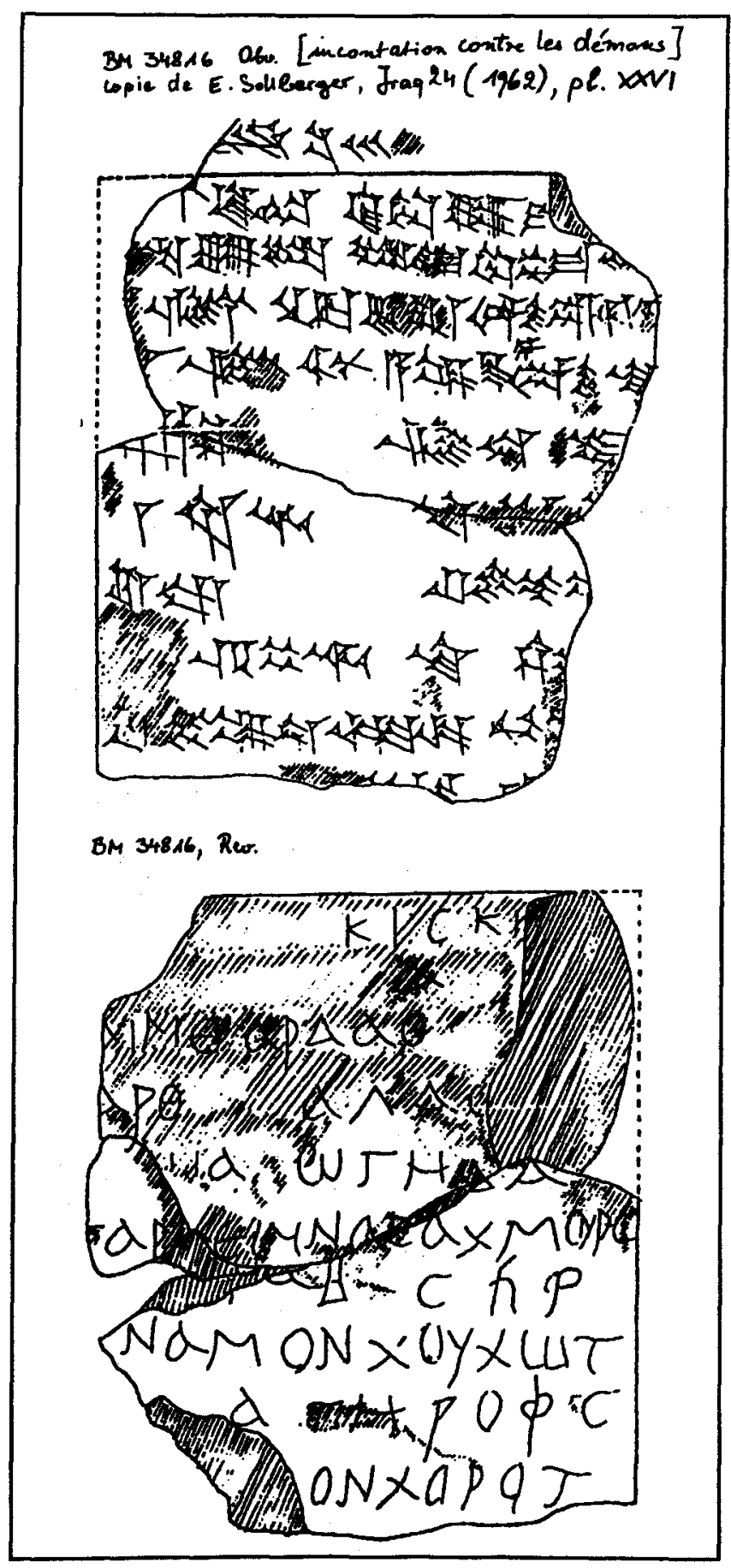

